\title{
GENERALIZED EQUILIBRIUM PROBLEMS IN ABSTRACT CONVEX SPACES
}

\author{
C.G. ALIZADEH, S.M. VAEZPOUR, AND A. PETRUŞEL
}

Received 08 December, 2014

\begin{abstract}
In this paper, the existence of solution for generalized equilibrium problems under compactness or non-compactness assumptions in abstract convex spaces is proved. The closedness and the convexity of the solution set are also obtained. Our results generalize and improve some recent results in the literature.
\end{abstract}

2010 Mathematics Subject Classification: 47H10; $49 \mathrm{~J} 40$.

Keywords: equilibrium problem, abstract convex space, $\Gamma$-convex, set-valued map, existence results

\section{INTRODUCTION}

Let $K$ be a convex subset of topological vector space $X$ and let $F: X \times X \rightarrow \mathbb{R}$ be a scalar bifunction such that $F(x, x) \geq 0$, for all $x \in K$. By an equilibrium problem, Blum and Oettli understood the problem of finding $\bar{x} \in K$ such that $F(\bar{x}, y) \geq 0$ for all $y \in K$, which is called equilibrium problem (for short EP). Many authors extended EP to vector case in different ways, see for example [4,9, 10,13].

The equilibrium problem contains many important problems like optimization, variational inequalities, minimax inequalities, Nash equilibrium problems, and complementarity problems. Recently, many researchers investigated different models of equilibrium problem and established general results on the existence of the equilibrium problem. For more details see [1-3,5-7, 11, 17].

In order to establish existence results, convexity plays an important role, which is a rather restrictive assumption. On the other hand, the concept of abstract convex spaces, which include convex subsets of topological vector space, C-spaces and Gconvex spaces, was introduced by Sehie Park [14] in 2006.

In this paper, using KKM theorem in abstract convex spaces, the convexity assumption is relaxed to $\Gamma$-convexity, in order to obtain existence of the solution of

For the third author, this work was made possible by the research grant GSCE offered by BabeşBolyai University Cluj-Napoca, No. 30248/22.01.2015. 
generalized equilibrium problem (for short GEP) in abstract convex space is presented. Our results extend and generalize various existence theorems for similar problems. Although in general the solution set fails to be convex, here a sufficient condition such that the solution set is to be convex is stated. A characterization of the closedness of the solution set is also given. Meanwhile, Theorem 3.1 of [10] is extended.

\section{PRELIMINARIES}

Let $X$ and $Y$ be topological vector spaces and $K$ be a convex subset of $X$. Let $F: K \times K \multimap Y$ be a set-valued function and $W \subseteq Y$. There are several possible ways to generalized equilibrium problems. The following problem was extensively studied:

$$
\text { Find } \bar{x} \in K \text { such that } F(\bar{x}, y) \subseteq W \quad \forall y \in Y \text {. }
$$

Consider a closed convex cone $C$ in $Y$. If $W=Y \backslash-$ int $C$ (where int $C \neq \varnothing$ ) then the generalized vector equilibrium problem may be formulated as one of the following three problems:

$$
(G V E P): \text { Find } \bar{x} \in K \text { such that } F(\bar{x}, y) \subseteq(Y \backslash-i n t C) \quad \forall y \in Y \text {, }
$$

or

$$
\text { Find } \bar{x} \in K \text { such that } F(\bar{x}, y) \not \subset-i n t C \quad \forall y \in Y \text {, }
$$

or

$$
\text { Find } \bar{x} \in K \text { such that } F(\bar{x}, y) \cap(Y \backslash-i n t C) \neq \varnothing \quad \forall y \in Y \text {. }
$$

Furthermore, if $W=C$ then the strong version of $G V E P$ is considered as follows:

$$
(S G V E P): \text { Find } \bar{x} \in X \text { such that } F(\bar{x}, y) \subseteq C \quad \forall y \in Y \text {, }
$$

$G V E P$ has been extensively studied by many authors in recent years (see $[1-3,5-7$, 11,17]).

We give now some notation and recall some definitions, which are intended to be used in the sequel.

Let $A$ be a subset of topological space $X$. We denote by $\bar{A}$ the closure of $A$ in $X$. Let $\langle D\rangle$ be the set of all nonempty finite subsets of a set $D$. A multimap (or a set-valued mapping) $F: X \multimap Y$ is a mapping $F: X \rightarrow 2^{Y}$ to the power set of $Y$, while $F^{-}: Y \multimap X$ is defined by $F^{-}(y):=\{x \in X \mid y \in F(x)\}$ for $y \in Y$.

An abstract convex space $(E, D ; \Gamma)$ (see [16]) consists of a topological space $E$, a nonempty set $D$ and a multimap $\Gamma:\left\langle D>\multimap E\right.$ with nonempty values $\Gamma_{A}:=\Gamma(A)$ for $A \in\langle D\rangle$. For any nonempty $D^{\prime} \subseteq D$, the $\Gamma$-convex hull of $D^{\prime}$ is denoted and defined by

$$
\operatorname{co}_{\Gamma} D^{\prime}:=\bigcup\left\{\Gamma_{A} \mid A \in<D^{\prime}>\right\} \subseteq E .
$$


A subset $X$ of $E$ is called a $\Gamma$ - convex subset of $(E, D ; \Gamma)$ relative to $D^{\prime}$ if for any $N \in<D^{\prime}>$, we have $\Gamma_{N} \subseteq X$, that is, $c o_{\Gamma} D^{\prime} \subseteq X$.

When $D \subseteq E$, a subset $X$ of $E$ is said to be $\Gamma$-convex if $\operatorname{co}_{\Gamma}(X \cap D) \subseteq X$; in other words, $X$ is $\Gamma$-convex relative to $D^{\prime}:=X \cap D$. In case $E=D$, let $(E ; \Gamma):=$ $(E, E ; \Gamma)$. If $E$ is compact, then $(E, D ; \Gamma)$ is called a compact abstract convex space.

It is obvious that any vector space $E$ is an abstract convex space with $\Gamma=c o$, where $c o$ is the convex hull in a vector space. In specially, $(\mathbb{R} ; c o)$ is an abstract convex space. Several examples of abstract convex spaces are given in [15] and the references therein.

Definition 1 ([16]). Let $(E, D ; \Gamma)$ be an abstract convex space. If $G: D \multimap E$ satisfies

$$
\Gamma_{A} \subseteq G(A):=\bigcup_{y \in A} G(y) \text { for all } A \in<D>,
$$

then $G$ is called a KKM map.

Definition 2 ([16]). The partial KKM principle for an abstract convex space $(E, D ; \Gamma)$ means that, for any closed-valued KKM mapping $G: D \multimap E$, the family $\{G(z)\}_{z \in D}$ has the finite intersection property. The KKM principle means that the same property also holds for any open-valued KKM map.

Definition 3. Let $(E, D ; \Gamma)$ be an abstract convex space. The set-valued mapping $G: D \multimap E$ is called:

(a) Intersectionally closed-valued if $\bigcap_{z \in D} \overline{G(z)}=\overline{\bigcap_{z \in D} G(z)}$;

(b) Transfer closed-valued if $\bigcap_{z \in D} \overline{G(z)}=\bigcap_{z \in D} G(z)$.

Luc et al. [12] noted that $(a) \Leftarrow(b)$ and gave examples of multimaps satisfying (a) but not $(b)$.

Definition 4 ([16]). For an abstract convex space $(E, D ; \Gamma)$, a subset $X$ of $E$ is said to be intersectionally closed (resp., transfer closed) if there is an intersectionally (respectively, transfer) closed-valued map $G: D \multimap E$ such that $X=G(z)$ for some $z \in D$.

We state now a theorem proved by Park in [15] which will be used in the main section.

Theorem 1 ([15], Generalized Partial KKM Principle). Let $(E, D ; \Gamma)$ be a compact abstract convex space satisfying the partial KKM principle and $G: D \multimap E a$ mapping such that:

(1) G is closed-valued; 
(2) $G$ is a KKM map.(that is, $\Gamma_{A} \subseteq G(A)$ for all $A \in<D>$ ).

Then

$$
\bigcap\{G(z) \mid z \in D\} \neq \varnothing \text {. }
$$

\section{MAIN RESULTS}

Let $(X ; \Gamma)$ be an abstract convex space, $Y$ be a topological space and $W \subseteq Y$. Suppose that $K$ is a nonempty $\Gamma$ - convex subset of $X$ and $F: K \times K \multimap Y$. We consider the system of generalized equilibrium problem:

$$
\text { Find } \bar{x} \in K \text { such that } F(\bar{x}, y) \subseteq W \quad \forall y \in Y .
$$

We give an existence theorem for the above generalized equilibrium problem in compact abstract convex space, which is a generalization of Theorem 3.1. in [10] (indeed, if $(K ; \Gamma)=\left(\mathbb{R}^{n} ; c o\right)$ and $F$ is a vector-valued function, then we get Theorem 3.1 in $[10])$.

Theorem 2. Let $(X ; \Gamma)$ be an abstract convex space satisfying the partial KKM principle and $K$ be a compact and $\Gamma$-convex subset of $X$. Let $Y$ be a topological space and $W: K \multimap Y$ be any set-valued mapping with nonempty values. Let $F$ : $K \times K \multimap Y$ be a set-valued mapping satisfying the following conditions:

(i) $F(x, x) \subseteq W(x)$, for every $x \in K$;

(ii) for all $x, y \in K, F(x, y) \subseteq W(x)$ implies $F(y, x) \subseteq-W(y)$;

(i i i ) for all $x \in K$, the set $\{y \in K \mid F(x, y) \subseteq-W(x)\}$ is closed;

(iv) for all $x \in K$, the set $\{y \in K \mid F(x, y) \nsubseteq W(x)\}$ is $\Gamma$-convex;

(v) for all $y \in K, F(x, y) \subseteq-W(x)$ for all $x \in K$ implies $F(y, x) \subseteq W(y)$ for all $x \in K$;

Then, the solution set to the problem

$$
\text { find } \bar{x} \in K \text { such that } F(\bar{x}, y) \subseteq W(\bar{x}) \text { for all } y \in K \text {, }
$$

and that of the problem

$$
\text { find } \bar{x} \in K \text { such that } F(y, \bar{x}) \subseteq-W(y) \text { for all } y \in K \text {, }
$$

are nonempty and closed. Moreover, they both coincide.

Proof. Set $G(y):=\{x \in K \mid-F(y, x) \subseteq W(y)\}$. For the beginning, we prove that $\Gamma(A) \subseteq G(A)$ for all $A \in<K>$. If there exists $N \in<K>$ such that $\Gamma(N) \nsubseteq G(N)$ then there exists $x \in \Gamma(N)$ and $x \notin G(N)$ ( in other word $x \notin G(y), \forall y \in N$ ). Thus $-F(y, x) \nsubseteq W(y), \forall y \in N$, which implies $F(x, y) \nsubseteq W(x)$ for all $y \in N$ by assumption (ii). So, $N \in<\{y \in K \mid F(x, y) \nsubseteq \nsubseteq W(x)\}>$. By assumption (iv), $\Gamma_{N} \subseteq\{y \in K \mid F(x, y) \nsubseteq W(x)\}$. Since $x \in \Gamma_{N}$, we have

$$
x \in\{y \in K \mid F(x, y) \nsubseteq W(x)\}
$$


or $F(x, x) \nsubseteq W(x)$ this contradicts $(i)$. This proves that $\Gamma(A) \subseteq G(A)$ for all $A \in<$ $K>$. Now we find $\bar{x} \in K$ such that

$$
\bar{x} \in \bigcap_{y \in K}\{x \in K \mid-F(y, x) \subseteq W(y)\} .
$$

Because of assumption (ii i ) each set $G(y)$ is closed. An application of the generalized partial KKM principle (see Theorem 1) yields the existence of $\bar{x} \in K$ such that $\bar{x} \in \bigcap_{y \in K} G(y)$, i. e, $F(y, \bar{x}) \subseteq-W(y)$ for all $y \in K$. In other words, the second problem has a solution. By the assumption $(v)$ we conclude that such a solution is also a solution of the first problem. Both solution sets coincide by $(i i)$. The closedness is a consequence of (i i i ).

Example 1. Let $E$ denote $\mathbb{R}^{2}$ with metric $d_{r}$ which defined as follow for any $x, y \in E$ :

$$
d_{r}(x, y)=\left\{\begin{array}{cc}
d(x, y) & \exists \lambda \in \mathbb{R}: x=\lambda y \\
d(x, 0)+d(0, y) \text { otherwise }
\end{array},\right.
$$

where $d$ denote the Euclidean metric and 0 denote the origin. If there exists $\lambda \in \mathbb{R}$ such that $x=\lambda y$, then segment joining $x$ and $y([x, y])$ is the geodesic segment, otherwise, union of the segments $[x, 0]$ and $[0, y]$ is the geodesic segment. So, $E$ is a complete $\mathbb{R}$-tree space.

Also, we recall that a subset $A$ in $\mathbb{R}$-tree space $E$ is convex if for any $x, y \in A$, all geodesic segments joining $x$ and $y$ are contained in $A$.

Let $\Gamma_{A}:=\operatorname{conv}(A)$ for each $A \in E$, where $\operatorname{conv}(A)$ is intersection of all closed convex subsets of the $\mathbb{R}$-tree $E$, that contains $A .(E, \Gamma)$ is an abstract convex space which satisfies the partial KKM principle, see [15].

Let $X=(E, \Gamma), Y=\mathbb{R}^{2}, K=\{(x, y) \in[-1,1] \times[-1,1] \mid x=y \vee x=-y\}$ and $W=\left\{(r, s) \in \mathbb{R}^{2} \mid r \geq 0 \vee s \geq 0\right\}$. Suppose $F: K \times K \multimap \mathbb{R}^{2}$ defined by

$$
\begin{gathered}
F\left(\left(x_{1}, y_{1}\right),\left(x_{2}, y_{2}\right)\right) \\
=\left\{\begin{array}{lc}
(-1,-1) & x_{1}, y_{1}<0 \text { and }\left(x_{2} \leq 0, y_{2} \geq 0 \text { or } x_{2} \geq 0, y_{2} \geq 0\right) \\
(1,1) & \left(x_{1} \leq 0, y_{1} \geq 0 \text { or } x_{1} \geq 0, y_{1} \geq 0\right) \text { and } x_{2}, y_{2}<0 \\
(0,0) & \text { otherwise }
\end{array} .\right.
\end{gathered}
$$

Then $F$ satisfies the assumptions of Theorem 2 and so the solution set of

$$
\text { " find } \bar{x} \in K \text { such that } F(\bar{x}, y) \subseteq W(\bar{x}) \text { for all } y \in K \text { ", }
$$

is nonempty and closed. In fact the solution set is

$$
\{(x, y) \in K \mid y=-x \vee y=x \geq 0\} .
$$

Note that we can not apply Theorem 3.1 in [10] for the set-valued $F$, because in spite of convexity of $K$ the set $\{y \in K \mid F(x, y) \nsubseteq W(x)\}$ in $(E, \Gamma)$, they are not convex in Euclidean space $\mathbb{R}^{2}$. 
To deal with the unbounded case, inspired by Theorem 5.1 in [16], we state now the following lemma.

Lemma 1. Let $(E, D ; \Gamma)$ be an abstract convex space satisfying the partial KKM principle and $G: D \multimap E$ is intersectionally closed-valued mapping. Suppose there exists a mapping $H: E \multimap E$ satisfying:

(i) For each $x \in E, x \in H(x)$;

(ii) For each $x \in E, \operatorname{co}_{\Gamma}\left(D \backslash G^{-}(x)\right) \subseteq E \backslash H^{-}(x)$.

Then

$$
\bigcap\{G(z) \mid z \in D\} \neq \varnothing .
$$

Proof. It is sufficient to show that ( $i$ ) and (ii) imply that $G$ is a KKM map. Suppose that there exists an $N \in<D>$ such that $\Gamma_{N} \nsubseteq G(N)$. That is, there exists an $x \in \Gamma_{N}$ such that $x \notin G(y)$ for all $y \in N$. In other words, $N \in<D \backslash G^{-}(x)>$. By (ii), $\Gamma_{N} \subseteq E \backslash H^{-}(x)$. Since $x \in \Gamma_{N}$, we have $x \notin H^{-}(x)$ or $x \notin H(x)$. This contradicts (i). Thus $G$ is a KKM mapping so $\Gamma_{N} \subseteq G(N) \subseteq \overline{G(N)}$ for any $N \in<D>$. Therefore, $\overline{G(N)}$ is a KKM mapping. Since $(E, D ; \Gamma)$ satisfies the partial KKM principle and $\bar{G}$ has closed values, the family $\{\overline{G(z)}\}_{z \in D}$ has the finite intersection property. Since $G$ is intersectionally closed-valued, we have that

$$
\overline{\bigcap_{z \in D} G(z)}=\bigcap_{z \in D} \overline{G(z)} \neq \varnothing .
$$

Since $\overline{\bigcap_{z \in D} G(z)} \neq \varnothing$ we get that $\bigcap\{G(z) \mid z \in D\} \neq \varnothing$, which proves the lemma.

Theorem 3. Let $(X ; \Gamma)$ be an abstract convex space satisfying the partial KKM principle, $K$ be a $\Gamma$-convex subset of $X$ and $Y$ be a topological space. Let $F$ : $K \times K \multimap Y$ be a set-valued map, $W$ be a nonempty subset of $Y$ and suppose that:

(i) $F(x, x) \subseteq W$, for every $x \in K$.

(i i ) For each $z \in K,\{y \in K \mid F(y, z) \subseteq W\}$ is intersectionally closed.

(i i i ) For every fixed $y \in K, c_{\Gamma}\{z \in K \mid F(y, z) \nsubseteq W\} \subseteq\{x \in K \mid F(x, y) \nsubseteq W\}$. Then, there exists a point $x_{0} \in K$ such that $F\left(x_{0}, y\right) \subseteq W$ for every $y \in K$.

Proof. For each $z \in K$, let $G(z):=\{y \in K \mid F(y, z) \subseteq W\}$ (we proof that $\left.\bigcap_{z \in K} G(z) \neq \varnothing\right)$. By $(i i), G(z)$ is intersectionally closed. For each $x \in K$, let $H(x):=\{y \in K \mid F(x, y) \subseteq W\}$. Then, (i) and (iii) imply conditions (i) and (ii) of Lemma 1 respectively. By $(i), x \in H(x)$ for every $x \in K$. By (i i i ),

$$
\operatorname{co}_{\Gamma}\left(D \backslash G^{-}(x)\right) \subseteq K \backslash H^{-}(x)
$$

So, by Lemma 1 , we get that

$$
\bigcap\{G(z) \mid z \in K\} \neq \varnothing .
$$


Theorem 4. Let $(X ; \Gamma)$ be an abstract convex space, $K$ be a $\Gamma$-convex subset of $X$ and $Y$ be a topological space. Let $F: K \times K \multimap Y$ be a set-valued mapping and $W$ be a nonempty subset of $Y$. If for each $z \in K,\{y \in K \mid F(y, z) \subseteq W\}$ be transfer closed-valued, then the solution set is closed.

Proof. Let $G(z):=\{y \in K \mid F(y, z) \subseteq W\}$. Then $G: K \multimap K$ is a transfer closed-valued mapping. So, we have

$$
\bigcap_{z \in K} \overline{G(z)}=\bigcap_{z \in K} G(z)
$$

Since $G$ also is an intersectionally closed-valued map, we have

$$
\bigcap_{z \in K} \overline{G(z)}=\overline{\bigcap_{z \in K} G(z)}
$$

So, we conclude that

$$
\bigcap_{z \in K} G(z)=\overline{\bigcap_{z \in K} G(z)}
$$

Therefore, the solution set is closed.

Motivated by Balaj and Lin [8], we define the following concept.

Definition 5. Let $X$ be a $\Gamma$-convex set in an abstract convex space and $Y$ be a convex set in a vector space. A mapping $F: X \multimap Y$ is said to be:

(a) quasiconvex if $F\left(x_{1}\right) \cap C \neq \varnothing$ and $F\left(x_{2}\right) \cap C \neq \varnothing$ then $F(\bar{x}) \cap C \neq \varnothing$ for all convex sets $C \subseteq Y, x_{1}, x_{2} \in X$ and for any $\bar{x} \in \operatorname{co}_{\Gamma}\left\{x_{1}, x_{2}\right\}$.

(b) quasiconcave if $F\left(x_{1}\right) \subseteq C$ and $F\left(x_{2}\right) \subseteq C$ then $F(\bar{x}) \subseteq C$ for all convex sets $C \subseteq Y, x_{1}, x_{2} \in X$ and for any $\bar{x} \in \operatorname{co}_{\Gamma}\left\{x_{1}, x_{2}\right\}$.

Then, we can prove the following result.

Theorem 5. Let $(X ; \Gamma)$ be an abstract convex space, $K$ be a $\Gamma$-convex subset of $X, Y$ be a vector space and $W$ be a convex subset of $Y$. Let $F: K \times K \multimap Y$ be a set-valued map. If $F(\cdot, y)$ is a quasiconcave mapping for all $y \in K$, then solution set is $\Gamma$-convex set.

Proof. If $x_{1}, x_{2} \in E_{w}:=\cap_{y \in K}\{x \in K \mid F(x, y) \subseteq W\}$ with $x_{1} \neq x_{2}$, then $F\left(x_{1}, y\right) \subseteq W$ and $F\left(x_{2}, y\right) \subseteq W$ for all $y \in K$. For any $y \in K$, the quasiconcaveness of $F(., y)$ implies $F\left(\operatorname{co}_{\Gamma}\left\{x_{1}, x_{2}\right\}, y\right) \subseteq W$. Hence, $\operatorname{co}_{\Gamma}\left\{x_{1}, x_{2}\right\} \subseteq E_{w}$. Since $x_{1}$ and $x_{2}$ were arbitrary, we obtain that

$$
\operatorname{co}_{\Gamma} E_{w}=\bigcup\left\{\Gamma_{A} \mid A \in<E_{w}>\right\} \subset E_{w} .
$$

This means that $E_{w}$ is convex. 


\section{REFERENCES}

[1] Q. Ansari and F. Flores-Bazán, "Recession methods for generalized vector equilibrium problems," J. Math. Anal. Appl., vol. 321, no. 1, pp. 132-146, 2006, doi: 10.1016/j.jmaa.2005.07.059.

[2] Q. Ansari, I. Konnov, and J. Yao, "Existence of a solution and variational principles for vector equilibrium problems," J. Optim. Theory Appl., vol. 110, no. 1, pp. 481-492, 2001, doi: 10.1023/A:1017581009670.

[3] Q. Ansari, I. Konnov, and J. Yao, "On generalized vector equilibrium problems," Nonlinear Anal., vol. 47, no. 1, pp. 543-554, 2001, doi: 10.1016/S0362-546X(01)00199-7.

[4] Q. Ansari, I. Konnov, and J. Yao, "Characterizations of solutions for vector equilibrium problems," J. Optim. Theory Appl., vol. 113, no. 3, pp. 435-447, 2002, doi: 10.1023/A:1015366419163.

[5] Q. Ansari, W. Oettli, and D. Schläger, "A generalization of vectorial equilibria,” Math. Methods Oper. Res., vol. 46, no. 2, pp. 147-152, 1997, doi: 10.1007/BF01217687.

[6] Q. Ansari, A. Siddiqi, and S. Wu, "Existence and duality of generalized vector equilibrium problems," J. Math. Anal. Appl., vol. 259, no. 1, pp. 115-126, 2001, doi: 10.1006/jmaa.2000.7397.

[7] Q. Ansari and J. Yao, "An existence result for the generalized vector equilibrium problem," Appl. Math. Lett., vol. 12, no. 1, pp. 53-56, 1999, doi: 10.1016/S0893-9659(99)00121-4.

[8] M. Balaj and L. Lin, "Equivalent forms of a generalized kkm theorem and their applications," Nonlinear Anal., vol. 73, no. 3, pp. 673-682, 2010, doi: 10.1016/j.na.2010.03.055.

[9] M. Bianchi, N. Hadjisavvas, and S. Schaible, "Vector equilibrium problems with generalized monotone bifunctions," J. Optim. Theory Appl., vol. 92, no. 3, pp. 527-542, 1997, doi: 10.1023/A:1022603406244.

[10] F. Flores-Bazán and F. Flores-Bazán, "Vector equilibrium problems under asymptotic analysis," $J$. Global Optim., vol. 26, no. 2, pp. 141-166, 2003, doi: 10.1023/A:1023048928834.

[11] I. Konnov and J. Yao, "Existence of solutions for generalized vector equilibrium problems," $J$. Math. Anal. Appl., vol. 233, no. 1, pp. 328-335, 1999, doi: 10.1006/jmaa.1999.6312.

[12] D. Luc, E. Sarabi, and A. Soubeyran, "Existence of solutions in variational relation problems without convexity," J. Math. Anal. Appl., vol. 364, no. 2, pp. 399-407, 2010, doi: 10.1016/j.jmaa.2009.10.040.

[13] W. Oettli and D. Schläger, "Existence of equilibria for monotone multivalued mappings," Math Methods Oper. Res., vol. 48, no. 2, pp. 219-228, 1998, doi: 10.1007/s001860050024.

[14] S. Park, "On generalizations of the kkm principle on abstract convex spaces," Nonlinear Anal. Forum, vol. 11, no. 1, pp. 66-77, 2006.

[15] S. Park, "The kkm principle in abstract convex spaces: equivalent formulations and applications," Nonlinear Anal., vol. 73, no. 4, pp. 1028-1042, 2010, doi: 10.1016/j.na.2010.04.029.

[16] S. Park, "New generalizations of basic theorems in the kkm theory," Nonlinear Anal., vol. 74, no. 9, pp. 3000-3010, 2011, doi: 10.1016/j.na.2011.01.020.

[17] I. Sadeqi and C. Alizadeh, "Existence of solutions of generalized vector equilibrium problems in reflexive banach spaces," Nonlinear Anal., vol. 74, no. 6, pp. 2226-2234, 2011, doi: 10.1016/j.na.2010.11.027.

Authors' addresses

\section{C.G. Alizadeh}

Department of Mathematics, Science and Research Branch, Islamic Azad University, Tehran, Iran

\section{S.M. Vaezpour}

Department of Mathematics and Computer Science,Amirkabir University of Technology, Hafez Ave., P.O. Box 15875-4413, Tehran, Iran

E-mail address: vaezeaut.ac.ir 


\section{A. Petruşel}

Department of Mathematics, Babeş-Bolyai University Cluj-Napoca,, Kogălniceanu Street No.1, 400084, Cluj-Napoca, Romania.

E-mail address: petruselemath.ubbcluj.ro 